\title{
Exploring Vital Sign Data Quality in Electronic Health Records with Focus on Emergency Care Warning Scores
}

Niclas Skyttberg ${ }^{1,2}$; Rong Chen²; Hans Blomqvist33; Sabine Koch²

${ }^{1}$ Capio St Görans Hospital, Stockholm; ${ }^{2}$ Health Informatics Centre Department of Learning, Informatics, Management, and Ethics, Karolinska Institutet, Stockholm; ${ }^{3}$ Department of Anaesthesia and Intensive Care Karolinska University Hospital, Stockholm, Sweden

\section{Keywords}

Vital signs, data quality, emergency medicine, electronic health records, clinical decision support systems.

\section{Summary}

Background: Computerized clinical decision support and automation of warnings have been advocated to assist clinicians in detecting patients at risk of physiological instability. To provide reliable support such systems are dependent on high-quality vital sign data. Data quality depends on how, when and why the data is captured and/or documented.

Objectives: This study aims to describe the effects on data quality of vital signs by three different types of documentation practices in five Swedish emergency hospitals, and to assess data fitness for calculating warning and triage scores. The study also provides reference data on triage vital signs in Swedish emergency care.

Methods: We extracted a dataset including vital signs, demographic and administrative data from emergency care visits ( $n=335027)$ at five Swedish emergency hospitals during 2013 using either completely paper-based, completely electronic or mixed documentation practices. Descriptive statistics were used to assess fitness for use in emergency care decision support systems aiming to calculate warning and triage scores, and data quality was described in three categories: currency, completeness and correctness. To estimate correctness, two further categories - plausibility and concordance - were used.

Results: The study showed an acceptable correctness of the registered vital signs irrespectively of the type of documentation practice. Completeness was high in sites where registrations were routinely entered into the Electronic Health Record (EHR). The currency was only acceptable in sites with a completely electronic documentation practice.

Conclusion: Although vital signs that were recorded in completely electronic documentation practices showed plausible results regarding correctness, completeness and currency, the study concludes that vital signs documented in Swedish emergency care EHRs cannot generally be considered fit for use for calculation of triage and warning scores. Low completeness and currency were found if the documentation was not completely electronic.

\section{Correspondence to:}

Niclas Skyttberg, MD

Health Informatics Centre

Department of Learning, Informatics, Management, and Ethics, Karolinska Institutet

17177 Stockholm, Sweden

Email: Niclas.skyttberg@ki.se

Phone +46700028774

\author{
Appl Clin Inform 2017; 8: 880-892 \\ https://doi.org/10.4338/ACI-2017-05-RA-0075 \\ received: 6. May 2017 \\ accepted in revised form: 3. July 2017 \\ published: August 30, 2017 \\ Citation: Skyttberg N, Chen R, Blomqvist H, Koch S. \\ Exploring Vital Sign Data Quality in Electronic Health \\ Records with Focus on Emergency Care Warning \\ Scores. Appl Clin Inform 2017; 8: 880-892 \\ https://doi.org/10.4338/ACI-2017-05-RA-0075
}




\section{Background and significance}

Early detection of patients at risk of sepsis or other physiological instabilities is known to improve clinical outcome [1]. In order to identify patients at risk, a number of scoring systems can be used, for example, RETTS [2], NEWS [3] or more specific diagnostic scoring systems for sepsis detection [1]. All scoring systems use vital signs for their calculation of warning scores [4]. The triage process and the clinical workflow in emergency wards usually include the measurement and documentation of vital signs ( Figure 1 ) $[5,6]$.

To assist clinicians in detecting patients at risk of physiological instability clinical decision support and automation of warnings have been advocated [7, 8]. Such systems have to rely on the data in the Electronic Health Record Systems (EHRs) to provide accurate warnings. Hence, data quality is essential, and can be defined as the capability of the data to be used effectively and rapidly to inform and evaluate decisions [9]. This capability is labelled fitness for use [10], and to assess data quality in EHRs Weiskopf and Weng propose a framework consisting of three dimensions: Completeness, Correctness and Currency [11].

Completeness may be defined as availability and accessibility of expected entries in the EHR. Vital signs are routinely measured [12], and EHRs are available at all Swedish emergency departments [13]. From those facts a high level of completed vital sign registrations may be expected. However, previous research at nine Swedish emergency departments showed that less than half of them document triage vital signs directly in the EHR [6]. Correctness refers to how true the data is. If the correctness cannot be directly measured surrogate measures like plausibility and concordance may be used to estimate correctness [11]. Plausibility is an estimate whether it seems reasonable to assume that the data are true while concordance compares agreement between corresponding data sets. The currency of the data deals with the temporal aspects of data quality. Many studies of EHR data quality have a perspective on retrospective reuse of data for research or storage in quality registries. From that perspective, the currency of the data may be of less importance. For real-time CDS, not least in acute medicine, the currency of the data is, however, of utmost importance Although there are some studies on vital sign data quality $[6,14,15]$, little is known about fitness for use for automatic calculations of warning scores and triage in the emergency care context.

\section{Objectives}

This study aims to describe the effects of different types of documentation practices on vital sign data quality, and to evaluate vital sign fitness for use in emergency care clinical decision support systems that provide calculations of warning and triage scores. The study presents a method for analysing vital sign data quality in EHRs and provides reference data on triage vital sign data in emergency care from five different Swedish hospitals.

\section{Methods}

\subsection{Data collection}

Data were extracted from the electronic health records of emergency departments at five different sites ( Table I). These sites were purposely selected to represent different documentation practices found in a previous qualitative study of factors affecting vital sign quality [6]. Three groups were formed according to the different types of documentation practices at the sites; paper-based documentation, mixed documentation and electronic documentation ( $>$ Table I). At two sites documentation was done on a structured paper-based template, and no entries of vital signs were routinely done in the EHR. Albeit EHRs were available at these two sites, they were not always used for documenting the vital signs measured immediately during triage. One site used a mixed approach where documentation was first done on a paper-based template and later transferred into the EHR. Finally, two sites had a fully electronic documentation practice where vital signs were entered directly into 
the EHR. No site made use of an automated registration of vital signs using medico-technical equipment.

Two different EHR systems, with four separate installations, were used at the five sites. The two paper-based sites and the mixed documentation practice site used EHR1, and all these three sites had separate installations of the system and the patient database. The two sites with a completely electronic documentation practice used the same installation of the EHR 2, and they also shared the same patient database. Although the installation was shared between the fully electronic sites, assigned clinical workflows and work practices varied between the sites. All emergency department visits at the five sites during 2013 were included in this study. An exclusion criterion was age $<18$ years. For each visit a specified dataset was extracted that included; patient age, gender, assigned clinical workflow (e.g. Surgery, Orthopedics, Internal Medicine), registered arrival time, saturation, systolic blood pressure, heart rate, temperature, respiratory rate and time of entry for the vital sign measurements.

\subsection{Data analysis}

Statistical analysis was done using SPSS Statistics (IBM, 2014) and Microsoft Excel (Microsoft Excel, 2016). As proposed by previous work on data quality in EHRs the data were evaluated regarding completeness, currency and correctness [11].

\subsection{Completeness}

Completeness was calculated for all vital signs, and the mean value of completeness for the sites is reported in the study. The completeness per vital sign was calculated by dividing the number of visits with a registration of the specified vital sign by the total number of visits.

\subsection{Correctness}

To directly measure correctness, the state of the patient at the time of measurement would be the golden standard for comparison, but because the study was performed retrospectively this was not known, and therefore, correctness was evaluated by the surrogate measures plausibility and concordance. Plausibility [11] can be defined as an estimate of how reasonable a data entry is in respect to the biological process it is supposed to represent, and in this study all non-valid data are treated as affecting the plausibility negatively. Data that was deemed not valid could be either not applicable or out of biological/clinical relevant range. Not applicable data could be reported in a wrong format, for example, strings of text were a number would be expected. Out of range data was defined by the researchers and discussed with senior consultants in emergency medicine at the sites ( Table II). For the definitions of out of range data the aim was to identify outliers from a clinical and biological perspective. The sum of outliers and non-valid data was identified and reported for the studied parameters. The mean value of the non-valid vital sign data per site is reported in the study. Plausibility was also assessed by studying the distributions of the data expecting normal biological distributions of the vital signs. Concordance [11] relates to whether data agreement exists between sources that aim to describe the same phenomenon, and in this study we compared agreement between the data sets in the sites. We used descriptive statistical data, boxplots and distribution plots to describe and analyse the concordance between the data sets of vital sign measurements. By evaluating both the plausibility and the concordance of the data we were able to assess the correctness of the vital sign data.

\subsection{Currency}

Triage including vital sign measurements is recommended to be performed within 15 minutes of arrival [12], and therefore this study was designed to relate the time of the vital sign registrations to the documented time of arrival in the EHR. The time of arrival is automatically set when the patient registers at the arrival desk in the emergency department. The time of arrival is considered robust and is used for quality measures and reporting in research, like in ED waiting times [16] and door to 
needle time in thrombolysis of stroke [17]. The time to registration of measurement was calculated by calculating the difference in minutes between the arrival time and the time of the closest registered vital signs. Descriptive statistics were used to evaluate the time to documentation. Negative time differences and differences of more than 240 minutes were considered non-valid.

\section{Results}

\subsection{Background information}

The demographic data ( $>$ Table I) show that the majority of patients (63-77\%) were assigned to the clinical workflow regarding internal medicine or surgically related complaints. All sites differentiate between surgical and internal medicine complaints except one site that has a common "single clinical workflow" for these patients.

\subsection{Completeness}

The level of completeness varied greatly between the sites $1-95 \%$ ( Table I). The group that used a paper-based documentation practice had the lowest completeness (1-2\%). Routines in the electronic documentation group did not include triage with vital sign measurements for all patients; patients assigned to the clinical workflow "other" were not triaged. These patients often had ear/nose/ throat related problems or complaints that were perceived to have lower acuity. Subgroup analysis in the electronic documentation group, with adjustment for the patients not expected to be triaged with vital signs increased the completeness to $85 \%$. This showed that completeness was acceptable for the sites using mixed or electronic documentation and that simple descriptive statistics can be used to assess completeness. There was a lack of standardized documentation routines for heart rate in the electronic documentation group. This made it complicated to retrieve data and resulted in low completeness for heart rate data in the group ( $>$ Table III).

\subsection{Correctness of vital sign measurements}

The descriptive statistical analysis of the registered vital sign values showed similarities between the groups ( $>$ Table III) ( $>$ Figure 2 ). Although there was overall concordance between the vital sign values there were deviations in the distributions of oxygen saturation in the paper-based documentation practice, where visual inspection of the distribution curve showed a lack of concordance and indicated lower data quality in the segment above $94 \%$. Further there was also some discord in the distributions of respiratory rate. The registrations of systolic blood pressure, respiratory rate and heart rate were affected by round-off practices. Systolic blood pressure had values aggregating at zero end digits and to a lesser degree at endings of five. Sub-group analysis of blood pressures between 105 and 115 showed that the registration of 110 was three times as frequent as any other registration in the interval, and that blood pressures wrongly rounded upwards to 110 constituted about $1,4 \%$ of all the measured blood pressures in the study. Similar patterns were evident for heart rate and respiratory rate, while the temperature registrations seemed to be unaffected by round off practices. Subgroup analysis of correctness depending on time of entry and assigned clinical workflow did not alter any of these patterns and did not seem to affect correctness. The non-valid data was low $(0.1 \%)$ in all groups. Thus the vital sign values gave an overall plausible impression. The round-off practices are accepted in the current work practice, and the discord in the respiratory rate and oxygen saturation was within the normal reference intervals and thus unlikely to affect the results of triage or warning score calculations. Overall, the results showed that the correctness was acceptable for use for automatic calculation of triage and warning scores.

\subsection{Currency}

The results indicate that in the mixed and electronic documentation practices about $50 \%$ of the measurements were registered in the EHR within 20 minutes and $75 \%$ within 37 and 29 minutes of 
arrival respectively. This contrasted the paper-based flow where the median time to documentation was 116 minutes. The difference was even more obvious when studying the boxplots and distribution curves of the time to documentation ( $>$ Figure 3 ), where a lack of normally distributed data showed that there was no robust standardized process for documenting the vital signs in the paperbased documentation practice. This was not unexpected as there were no routines for documenting the vital signs in the EHR in this group. In all groups, the time to documentation varied over the time of day. Figure 4 describes the percentage of patient registrations and the mean time from arrival to vital sign documentation throughout the day. In the mixed and paper-based documentation groups, there was clear covariation with the number of patient registrations over the time of the day ( Figure 4). This indicated that vital sign documentation was delayed when the demand of measurements was high and that the time to documentation was more affected in the mixed documentation group than in the electronic documentation group. In the paper-based and mixed documentation groups, there were high levels of invalid data (33 vs 47\%). Further analysis showed that these vital signs were usually documented through dictation by doctors and entered into the EHR much later by secretaries. When the secretaries registered the vital signs, the time was set to the time of patient arrival resulting in a zero-time difference. This affected the plausibility of the paper-based and mixed documentation time differences. Only the electronic documentation practice resulted in high currency of the documented vital signs. However, even when using electronic documentation practices, less than $50 \%$ of the registrations were available within 15 minutes, which is the recommended time for triage in emergency departments. Thus the relationship between the time of measurement and the time of arrival turned out to be a relevant indicator of vital sign currency.

\section{Discussion}

\subsection{Completeness}

To be used for automatic calculation of warning scores the vital signs have to be registered in the EHR. Because most Swedish sites use a triage system to prioritize the arriving patients, a high level of vital sign measurements is expected. Although all studied groups had EHRs implemented at the emergency departments, the results show that they are not uniformly used for registrations of vital signs. In this study completeness was high in sites and assigned clinical workflows where department routines stated that documentation should be done in the EHR. This supports findings that completeness may be improved by standardizing the documentation $[6,15]$. The variability within and between sites may be attributed to EHR implementation barriers. A switch from a paper-based documentation practice needs leadership to overcome resistance to change [18]. In sites using electronic documentation, completeness may be improved by quality improvement programs [14]. Findings from this study show that completeness can be evaluated by descriptive studies. Before a triage CDSS can be implemented the completeness of the vital sign recordings has to be ascertained because there seems to be variability between sites and even within sites depending on the assigned clinical workflow.

\subsection{Correctness}

When comparing the datasets with descriptive statistics there are similarities between distributions. This was interpreted as a high concordance between the data sets, and the finding is in line with other studies of vital sign correctness in EHRs [19]. The discord found in respiratory rate may signal a clinical challenge in respiratory rate measurement [20]. Measuring respiratory rate is known to be complicated because it is time-consuming, and a manual observation has to be done while the patient is unaware of what measurement is occurring. We suspect that for patients with a perceived as normal respiration rate the RR is set to 14 in some sites and 16 in other instead of staff actually taking an exact measurement. The discord in oxygen saturation above $94 \%$ in the paper-based group may indicate a tendency to deem values above $94 \%$ as normal saturation and rounding them off upwards, either to 98 or $100 \%$. 
Despite the overall impression of stable quality in the correctness, there were findings showing that round-off practice is present in all sites. Rounding off with a preference to zero end digits is described in multiple studies of blood pressure measurements [21]. The clinical impact of the roundoff practice is not clear, but other studies have warned that zero end digit preferences may affect the likelihood of eligibility for drug treatment in hypertension [21]. This finding may be important when applying decision rules based on registered vital signs. In the NEWS scale a trigger for low systolic blood pressure is set to below $111 \mathrm{~mm} / \mathrm{hg}$ [3]. Sub-group analysis shows an end-digit preference that would shift a number of patients from the normal cohort to the low blood pressure cohort. In the study at least $1,5 \%$ of the patients would be shifted to a higher national early warning score considering blood pressure. However, this practice seems to be accepted, and at present decisions are likely to be on rounded data. From this perspective, the data can be considered fit for use in the current state. This study shows that the correctness of the vital signs is fit for use in calculations of warning scores, triage, and research.

\subsection{Currency}

Time is important when calculating triage and early warning scores. In this study we used the time of arrival and the time of documentation to assess currency of the vital sign registrations. Because the use case in this study was calculation of triage and warning scores, the time it takes from arrival until the automatic calculations can be made is critical. The shorter the time, the higher the currency. Findings in this study indicate that currency is higher in the sites with electronic documentation practice. In these sites, data show that $75 \%$ of the measurements are registered within 30 minutes of arrival, which seems clinically relevant. We and other groups have earlier shown that variability in manual vital sign documentation will impact currency and completeness of the vital signs $[6,15]$. In this study using a paper-based template to record vital signs lead to a delay of entry in the EHR and invalid time stamps. According to our results a manual system seems to fare progressively worse with increased workload compared to a more electronic documentation practice. This is in line with other studies that have shown that the currency of the registered vital signs may be improved by facilitating point of care documentation and/or automatized registrations by medical devices [22-24]. None of the studied sites had EHR integrations with medical devices for vital sign measurements, and it is interesting to note that the staff may perceive that such integrations may reduce patient contact and even influence correctness negatively $[6,25]$. Even if such risk is addressed by human validation of the entries, there are still challenges with all aspects of interoperability, technical, operational, and semantical that need to be overcome before auto-entry will be standard in the emergency care process. We showed that currency of the vital sign recordings might be studied by plotting the distributions of the time to measurement, and further our results showed that the currency of the vital sign cannot be considered generally fit for use in CDSS or clinical research focusing on the time of measurement. This finding is in line with other studies that have shown systematic errors in the timestamps in EHR vital sign data [22, 26].

\subsection{Summary of discussion}

In table IV we have summarized the effects of documentation practice on data quality. To be fit for use in clinical decision support used for calculation of emergency care warning scores, vital signs need to be correct, complete and available at the right time. This study shows that for paper-based and mixed documentation practices the currency of the vital signs will not suffice to give timely warnings during all hours of the day. Earlier studies indicate that less than half of the Swedish emergency departments have a fully electronic documentation practice for vital signs [6]. We conclude that vital signs in emergency care EHRs cannot generally be considered fit for use in emergency care clinical decision support systems.

\subsection{Limitations}

The main limitation of the study is the retrospective perspective. As the time of measurement was not known we have used the time from arrival to the time of vital sign documentation to study the 
currency of the vital signs. Since triage ideally is expected to be performed within 15 minutes of arrival, the time to documentation is a relevant measure because this is the earliest point in time that vital signs are available for a CDSS using EHR data.

\section{Conclusion}

Before emergency department vital signs can be reused for automatic calculation of triage and warning scores, data quality has to be ascertained and improved. Additional effects of improving and automatizing the documentation practice could be that staff workload decreases and more focus may be directed towards giving rather that documenting care. We showed that currency, completeness, plausibility and concordance of vital signs can be evaluated by descriptive statistics and comparison of multiple data sets. We provided reference vital sign data from five emergency departments for such use. We showed that electronic documentation of vital signs can result in acceptable data quality for reuse in the calculation of triage and warning scores. Using mixed documentation or paper-based documentation, however, will result in inadequate currency and completeness.

\section{Implications for clinical work and research}

This study shows that Swedish emergency care vital sign data cannot be considered generally fit for reuse in real time CDSS. This is of importance to clinicians aiming to develop alerting systems based on EHR vital signs in emergency care. Further studies on evaluating and improving vital sign data quality, especially the currency aspects, are encouraged.

\section{Multiple Choice Questions}

1. What influences vital sign data quality in emergency care electronic health records?

a. Time of the day when the emergency care visit takes place

b. Documentation practice

c. The number of physicians on duty

d. The brand of the EHR system

Answer b. is correct. Currency and Completeness will be affected by the documentation practice. Time of the day seems to affect the time to documentation but the effect is moderate in this study. We could not observe any difference between the different EHR systems used in this study on vital sign data quality.

2. Which data quality aspect is directly related to electronic documentation or data capture of vital signs?

a. Currency

b. Correctness

c. Plausibility

d. Concordance

Answer a. is correct. At least in our study currency of vital sign data was only achieved in completely electronic documentation practice.

\section{Conflict of interest}

NS is employed as Chief Medical Officer at one of the study sites. RC is employed as the Chief Medical Informatics Officer at Cambio Health Care Systems. Cambio Healthcare Systems is an Electronic Health Record provider to three of the studied sites. 


\section{Ethics approval}

The study was approved by the Stockholm region ethical committee (Dnr 2014/1207-31/4) and performed with anonymized data.

\section{Human subjects' protection}

As the data was anonymized and retrieved retrospectively no consent was obtained. Using anonymized data protected the integrity of the participants.

\section{Availability of data and material}

All data generated or analysed during this study are included in this published article [supplementary file 1].

\section{Authors' contributions}

All authors contributed to the work in a way that fulfils all of the four ICMJE criteria for authorship, including reading and approving the final manuscript. NS was the project leader, coordinated the study and was involved equally in all parts. RC contributed to the analysis of the results, drafting and reviewing of the manuscript. HB supported the work by feedback on results and analysis and critically reviewing of the manuscript. SK acted as project owner of the study contributing with scientific methods, drafting and reviewing of the manuscript. 


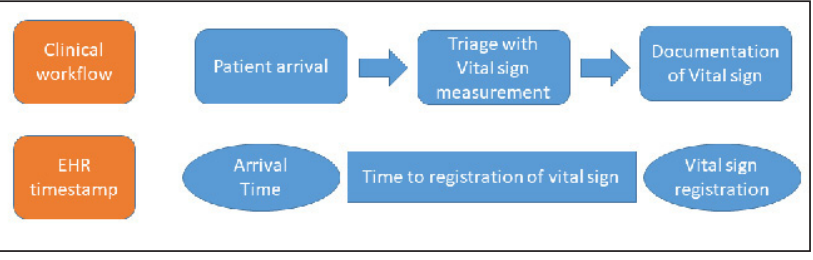

Fig. 1 Vital Sign Documentation Process

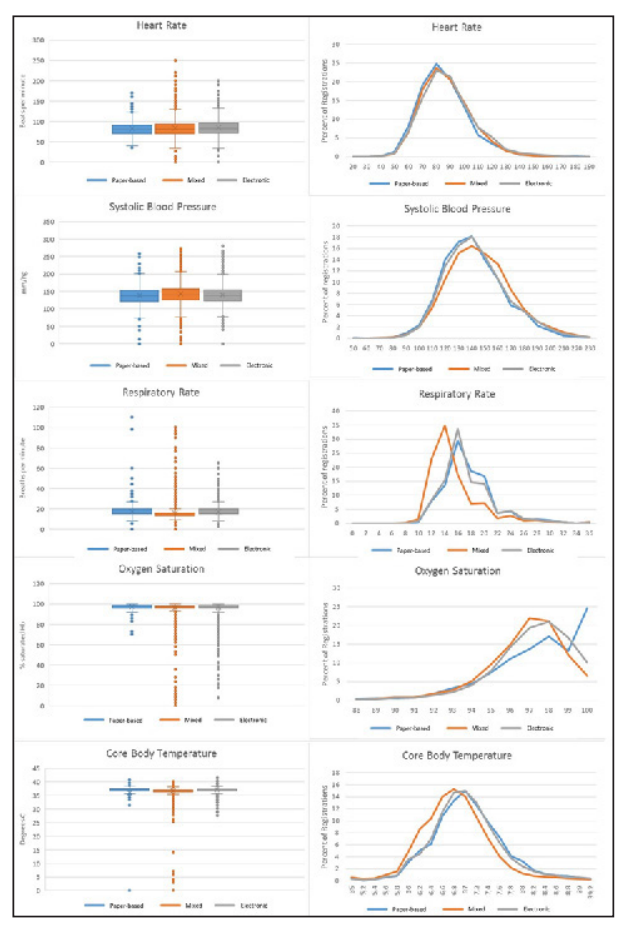

Fig. 2 Vital Sign Data Boxplots and Distribution

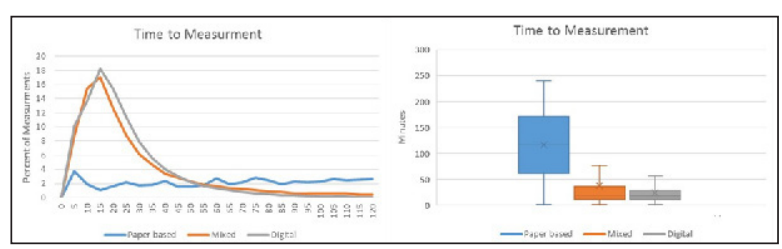

Fig. 3 Currency Distribution and Boxplot

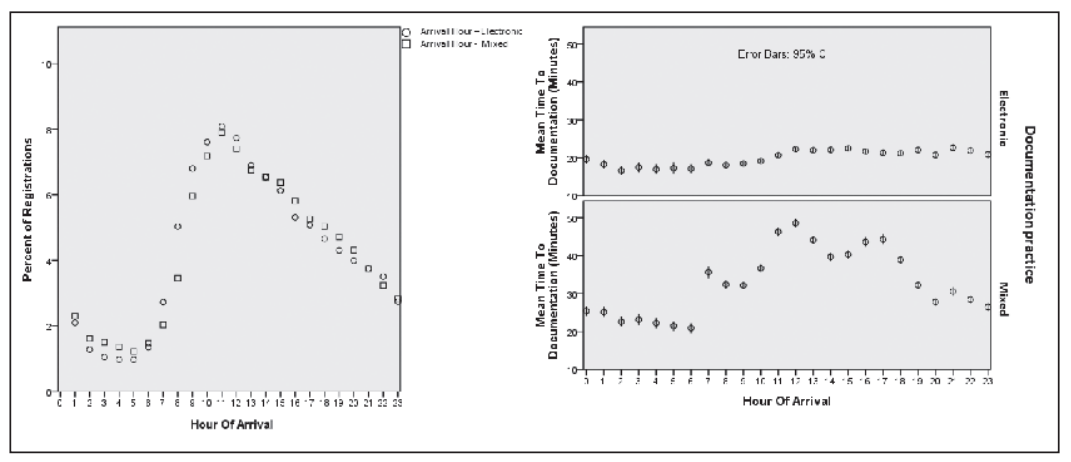

Fig. 4

Percent of registered arriving patients and time to vital sign documentation related to the time of day 
Table 1 Demographic data, Completeness and non-valid registrations

\begin{tabular}{|l|l|l|l|l|l|}
\hline & Site 1 & Site 2 & Site 3 & Site 4 & Site 5 \\
\hline n Visits & 59900 & 59679 & 62764 & 78991 & 73693 \\
\hline Age Mean & 54 & 54 & 45 & 51 & 50 \\
\hline Male/Female & $47 / 53$ & $50 / 50$ & $50 / 50$ & $44 / 56$ & $50 / 50$ \\
\hline EHR system & EHR 1 & EHR 1 & EHR 1 & EHR 2 & EHR 2 \\
\hline Installation of EHR & Inst A & Inst B & Inst C & Inst D & Inst D \\
\hline Documentation practice & Mixed & Paper-based & Paper-based & Electronic & Electronic \\
\hline Clinical workflow, \% & of visits & & & & \\
\hline Internal medicine & $48 \%$ & $46 \%$ & $45 \%$ & $40 \%$ & - \\
\hline Surgery & $28 \%$ & $31 \%$ & $30 \%$ & $23 \%$ & - \\
\hline One flow & - & - & - & - & $52 \%$ \\
\hline Orthopaedics & $24 \%$ & $20 \%$ & $18 \%$ & $15 \%$ & $12 \%$ \\
\hline Other & - & $3 \%$ & $7 \%$ & $22 \%$ & $36 \%$ \\
\hline Extracted data & & & & & $6.1 \%$ \\
\hline Completeness & $95 \%$ & $2 \%$ & $0.1 \%$ & $0.1 \%$ & $0.1 \%$ \\
\hline Non-valid data & $0.3 \%$ & $0.1 \%$ & & $71 \%$ & \\
\hline
\end{tabular}

\begin{tabular}{|l|l|}
\hline Vital Sign & Validity range \\
\hline Saturation $\mathrm{SPO}_{2}$ & $70-100 \%$ \\
\hline Systolic Blood Pressure & $60-240 \mathrm{~mm} / \mathrm{Hg}$ \\
\hline Heart Rate & $20-250 \mathrm{bpm}$ \\
\hline Temperature & $33-42 \mathrm{C}^{\circ}$ \\
\hline Respiratory Rate & $4-45$ breaths per minute \\
\hline Time to registered vital sign & $0-240$ min \\
\hline
\end{tabular}

Table 2 Valid vital sign range 
Table 3 Descriptive statistics of vital sign values

\begin{tabular}{|l|l|l|l|l|l|l|l|l|l|}
\hline Vital Sign & Documentation & $\mathbf{n}$ & Mean & Median & SD & Q1 & Q3 & Min & Max \\
\hline Heart Rate & Paper based & 2316 & 82,2 & 80 & 18,1 & 70 & 94 & 36 & 250 \\
& Mixed & 56559 & 83,3 & 81 & 18,3 & 70 & 91 & 0 & 175 \\
\hline & Electronic & 4133 & 84,9 & 82 & 19,7 & 71 & 96 & 0 & 200 \\
\hline Systolic Blood & Paper based & 3439 & 139,1 & 137 & 24,1 & 121 & 153 & 13 & 260 \\
\hline Pressure & Mixed & 58673 & 142,7 & 140 & 25,3 & 125 & 158 & 0 & 273 \\
\hline & Electronic & 107483 & 140,4 & 138 & 24,4 & 123 & 154 & 0 & 286 \\
\hline Respiratory Rate & Paper based & 2577 & 17,7 & 16 & 4,6 & 15 & 20 & 0 & 110 \\
\cline { 2 - 7 } & Mixed & 56490 & 15,6 & 14 & 5,1 & 13 & 16 & 0 & 100 \\
\hline & Electronic & 95216 & 17,3 & 16 & 4,2 & 15 & 20 & 3 & 65 \\
\hline Oxygen Saturation & Paper based & 1603 & 97,3 & 98 & 2,8 & 96 & 99 & 70 & 100 \\
\cline { 2 - 7 } & Mixed & 57785 & 96,5 & 97 & 3,8 & 96 & 98 & 0 & 100 \\
\hline & Electronic & 105320 & 97,0 & 97 & 2,7 & 96 & 99 & 8 & 100 \\
\hline Core Body Tem- & Paper based & 2741 & 37,0 & 37 & 0,7 & 36,6 & 37,4 & 31,4 & 40,8 \\
\hline perature & Mixed & 56112 & 36,8 & 36,8 & 0,9 & 36,4 & 37,1 & 0 & 41 \\
\hline & Electronic & 101253 & 37,0 & 36,9 & 0,7 & 36,6 & 37,3 & 27,6 & 41,9 \\
\hline Time to Regis- & Paper based & 3079 & 116 & 116 & 62 & 62 & 171 & 1 & 240 \\
\hline tration & Mixed & 58673 & 38,3 & 19 & 326 & 11 & 37 & 1 & 240 \\
\hline & Electronic & 113199 & 24,2 & 18 & 90 & 11 & 29 & 1 & 240 \\
\hline
\end{tabular}

\begin{tabular}{|l|l|l|l|l|l|} 
& Paper-based & Mixed & Electronic & $\begin{array}{c}\text { Table } 4 \\
\text { quality }\end{array}$ & Effects of documentation practice on data \\
\hline Completeness & Low & High & High & \\
\hline Correctness & High & High & High & \\
\hline Currency & Low & Low & Medium & \\
\hline
\end{tabular}




\section{References}

1. Dellinger RP, Levy MM, Rhodes A, Annane D, Gerlach H, Opal SM, Sevransky JE, Sprung CL, Douglas IS, Jaeschke R, Osborn TM, Nunnally ME, Townsend SR, Reinhart K, Kleinpell RM, Angus DC, Deutschman CS, Machado FR, Rubenfeld GD, Webb S, Beale RJ, Vincent J-L, Moreno R. Surviving Sepsis Campaign: international guidelines for management of severe sepsis and septic shock, 2012. Intensive Care Med 2013; 39: 165-228.

2. Pérez N, Nissen L, Nielsen RF, Petersen P, Biering K. The predictive validity of RETTS-HEV as an acuity triage tool in the emergency department of a Danish Regional Hospital. Eur J Emerg Med 2016; 23: 33-7.

3. National Early Warning Score (NEWS) National Early Warning Score (NEWS). 2012 (July). https://www. rcplondon.ac.uk/projects/outputs/national-early-warning-score-news. Last accessed on: June 3, 2017.

4. Churpek MM, Adhikari R, Edelson DP. The value of vital sign trends for detecting clinical deterioration on the wards. Resuscitation 2016; 102: 1-5.

5. Farrohknia N, Castrén M, Ehrenberg A, Lind L, Oredsson S, Jonsson H, Asplund K, Göransson KE. Emergency department triage scales and their components: a systematic review of the scientific evidence. Scand J Trauma Resusc Emerg Med 2011; 19: 42.

6. Skyttberg N, Vicente J, Chen R, Blomqvist H, Koch S. How to improve vital sign data quality for use in clinical decision support systems? A qualitative study in nine Swedish emergency departments. BMC Med Inform Decis Mak 2016; 16: 61.

7. Dean NC, Jones BE, Jones JP, Ferraro JP, Post HB, Aronsky D, Vines CG, Allen TL, Haug PJ. Impact of an Electronic Clinical Decision Support Tool for Emergency Department Patients With Pneumonia. Ann Emerg Med 2015; 66: 511-520.

8. Alsolamy S, Al Salamah M, Al Thagafi M, Al-Dorzi HM, Marini AM, Aljerian N, Al-Enezi F, Al-Hunaidi F, Mahmoud AM, Alamry A, Arabi YM. Diagnostic accuracy of a screening electronic alert tool for severe sepsis and septic shock in the emergency department. BMC Med Inform Decis Mak 2014; 14: 105.

9. Karr AF, Sanil AP, Banks DL. Data quality: A statistical perspective. National Institute of Statistical Sciences. https://www.niss.org/sites/default/files/technicalreports/tr129.pdf Last accessed: June 3, 2017

10. Juran JM, Gryna FM. McGraw-Hill: Juran’s Quality Control Handbook 1988.

11. Weiskopf NG, Weng C. Methods and dimensions of electronic health record data quality assessment: enabling reuse for clinical research. J Am Med Inform Assoc 2013; 20: 144-51.

12.SBU - Swedish Council on Health Technology Assessment. Triage Och Flödesprocesser På Akutmottagningen. En Systematisk Litteraturöversikt. Stockholm; 2010. http://www.sbu.se/sv/publikationer/SBU-ut varderar/triage-och-flodesprocesser-pa-akutmottagningen/. Last accessed on: June 13, 2017

13. Jerlvall L, Pehrsson T. eHealth in the county councils 2017. 2014; 1: 1-62.

14. di Martino P, Leoli F, Cinotti F, Virga A, Gatta L, Kleefield S, Melandri R. Improving vital sign documentation at triage: an emergency department quality improvement project. J Patient Saf 2011; 7: 26-9.

15. Miltner RS, Johnson KD, Deierhoi R. Exploring the frequency of blood pressure documentation in emergency departments. J Nurs Scholarsh 2014; 46: 98-105.

16. Wiler JL, Welch S, Pines J, Schuur J, Jouriles N, Stone-Griffith S. Emergency department performance measures updates: proceedings of the 2014 emergency department benchmarking alliance consensus summit. Acad Emerg Med 2015; 22: 542-53.

17.Sapkota B, Sirelkhatim A, Devlin T, Pitiyanuvath N, Dellinger C, Fesmire F. Reducing Door-To-Needle Times for Treatment of Acute Ischemic Stroke: Quality Improvement Initiative at a High Volume Comprehensive Stroke Center (P4.309). Neurology 2015; 84(14_Supplement): P4.309.

18. Boonstra A, Broekhuis M. Barriers to the acceptance of electronic medical records by physicians from systematic review to taxonomy and interventions. BMC Health Serv Res 2010; 10: 231.

19. Genes N, Chandra D, Ellis S, Baumlin K. Validating emergency department vital signs using a data quality engine for data warehouse. Open Med Inform J 2013; 7: 34-9.

20.Philip K, Richardson R, Cohen M. Staff perceptions of respiratory rate measurement in a general hospital. Br J Nurs 2013; 22: 570-4.

21. Broad J, Wells S, Marshall R, Jackson R. Zero end-digit preference in recorded blood pressure and its impact on classification of patients for pharmacologic management in primary care - PREDICT-CVD-6. Br J Gen Pract 2007; 57: 897-903.

22. Wager KA, Schaffner MJ, Foulois B, Swanson Kazley A, Parker C, Walo H. Comparison of the quality and timeliness of vital signs data using three different data-entry devices. Comput Inform Nurs 2010; 28: 205-12.

23. Smith LB, Banner L, Lozano D, Olney CM, Friedman B. Connected care: reducing errors through automated vital signs data upload. Comput Inform Nurs 2011; 27: 318-23. 
24. Dykes PC, Benoit A, Chang F, Gallagher J, Li Q, Spurr C, McGrath EJ, Kilroy SM, Prater M. The feasibility of digital pen and paper technology for vital sign data capture in acute care settings. AMIA. Annu Symp proceedings AMIA Symp 2006: 229-33.

25. Prgomet M, Cardona-Morrell M, Nicholson M, Lake R, Long J, Westbrook J, Braithwaite J, Hillman K. Vital signs monitoring on general wards: clinical staff perceptions of current practices and the planned introduction of continuous monitoring technology. Int J Qual Heal Care 2016; 28: 515-521.

26. Ward MJ, Froehle CM, Hart KW, Lindsell CJ. Operational data integrity during electronic health record implementation in the ED. Am J Emerg Med 2013; 31: 1029-33. 